\title{
Antidepressant medications $v$. cognitive therapy in people with depression with or without personality disorder
}

Jay C. Fournier, Robert J. DeRubeis, Richard C. Shelton, Robert Gallop, Jay D. Amsterdam and Steven D. Hollon

\section{Background}

There is conflicting evidence about comorbid personality pathology in depression treatments.

\begin{abstract}
Aims
To test the effects of antidepressant drugs and cognitive therapy in people with depression distinguished by the presence or absence of personality disorder.
\end{abstract}

\section{Method}

Random assignment of 180 out-patients with depression to 16 weeks of antidepressant medication or cognitive therapy. Random assignment of medication responders to continued medication or placebo, and comparison with cognitive therapy responders over a 12-month period.

\section{Results}

Personality disorder status led to differential response at 16 weeks; $66 \%$ v. $44 \%$ (antidepressants v. cognitive therapy respectively) for people with personality disorder, and $49 \% \mathrm{~V}$.
$70 \%$ (antidepressants $v$. cognitive therapy respectively) for people without personality disorder. For people with personality disorder, sustained response rates over the 12-month follow-up were nearly identical (38\%) in the prior cognitive therapy and continuation-medication treatment arms. People with personality disorder withdrawn from medication evidenced the lowest sustained response rate (6\%). Despite the poor response of people with personality disorder to cognitive therapy, nearly all those who did respond sustained their response.

\section{Conclusions}

Comorbid personality disorder was associated with differential initial response rates and sustained response rates for two well-validated treatments for depression.

\section{Declaration of interest}

R.C.S has received grant support from GlaxoSmithKline Pharmaceuticals. Funding detailed in Acknowledgements.
Although current American Psychiatric Association treatment guidelines state that 'cognitive behavioral therapy may be more effective than other treatments for depressed individuals with personality disorders, ${ }^{1}$ this statement appears to be largely based on a misunderstanding of data from the Treatment of Depression Collaborative Research Program. ${ }^{2,3}$ The results from this project did not reveal a personality disorder $\times$ treatment interaction, but rather a non-significant trend whereby people with no comorbid personality disorder responded more poorly to cognitive therapy than did people with personality disorder. Subsequent studies have not supported the claim that the presence of personality disorder predicts favourable response to cognitive therapy. ${ }^{4}$ Moreover, the conclusions from two recent meta-analyses reflect the controversy regarding whether comorbid personality pathology affects response to treatment for depression. $^{5,6}$ One reported that people with depression and comorbid personality disorder experienced poorer response when receiving either cognitive therapy or pharmacotherapy. ${ }^{6}$ The other, which included only trials of antidepressant medication, reported no difference in response as a function of personality pathology. ${ }^{5}$

We present data drawn from a multi-site randomised controlled trial comparing cognitive therapy and paroxetine for individuals diagnosed with moderate-to-severe depression. ${ }^{7,8} \mathrm{We}$ focus on whether the presence of comorbid personality disorder predicts differential response to cognitive therapy and pharmacotherapy, and we explore the effect of comorbid personality disorder on relapse once treatment is terminated.

\section{Method}

The sample characteristics, treatment protocols and main treatment outcome findings have been reported elsewhere. ${ }^{7,8}$ Briefly, the sample consisted of 240 out-patients with depression (measured using the Structured Clinical Interview for DSM-IV Diagnosis $)^{9}$ who registered a score of 20 or higher on the modified 17-item version of the Hamilton Rating Scale for Depression (HRSD). ${ }^{10}$ Personality pathology was assessed at intake using the Structured Clinical Interview for DSM-III-R Personality Disorders. ${ }^{11}$ Among the entire sample, $48 \%$ of individuals met criteria for at least one comorbid personality disorder. As was done in the Treatment of Depression Collaborative Research Program, participants with antisocial $(n=3)$ and schizotypal $(n=1)$ personality disorders were excluded from the trial. ${ }^{2}$ Those meeting criteria for borderline personality disorder $(n=8)$ were also excluded. The treatments under investigation were judged to be either ill-suited or too brief for individuals with depression comorbid with any of these three disorders. The distributions of personality disorders (shown in the online table DS1) were similar between the treatment arms and resemble those found in other samples of out-patients with depression. ${ }^{12}$ The institutional review boards of the University of Pennsylvania and Vanderbilt University approved the study's protocols. All participants provided written informed consent.

\section{Treatment}

Participants were randomly assigned to receive cognitive therapy $(n=60)$, antidepressant treatment with paroxetine $(n=120)$ or pill placebo $(n=60)$ (Fig. 1). After 8 weeks of the 16 -week acute treatment phase, the placebo arm was terminated and individuals who had been receiving placebo were offered antidepressant treatment at no cost. Because 8 weeks of treatment is generally not regarded as sufficient to treat people with comorbid personality disorders, 


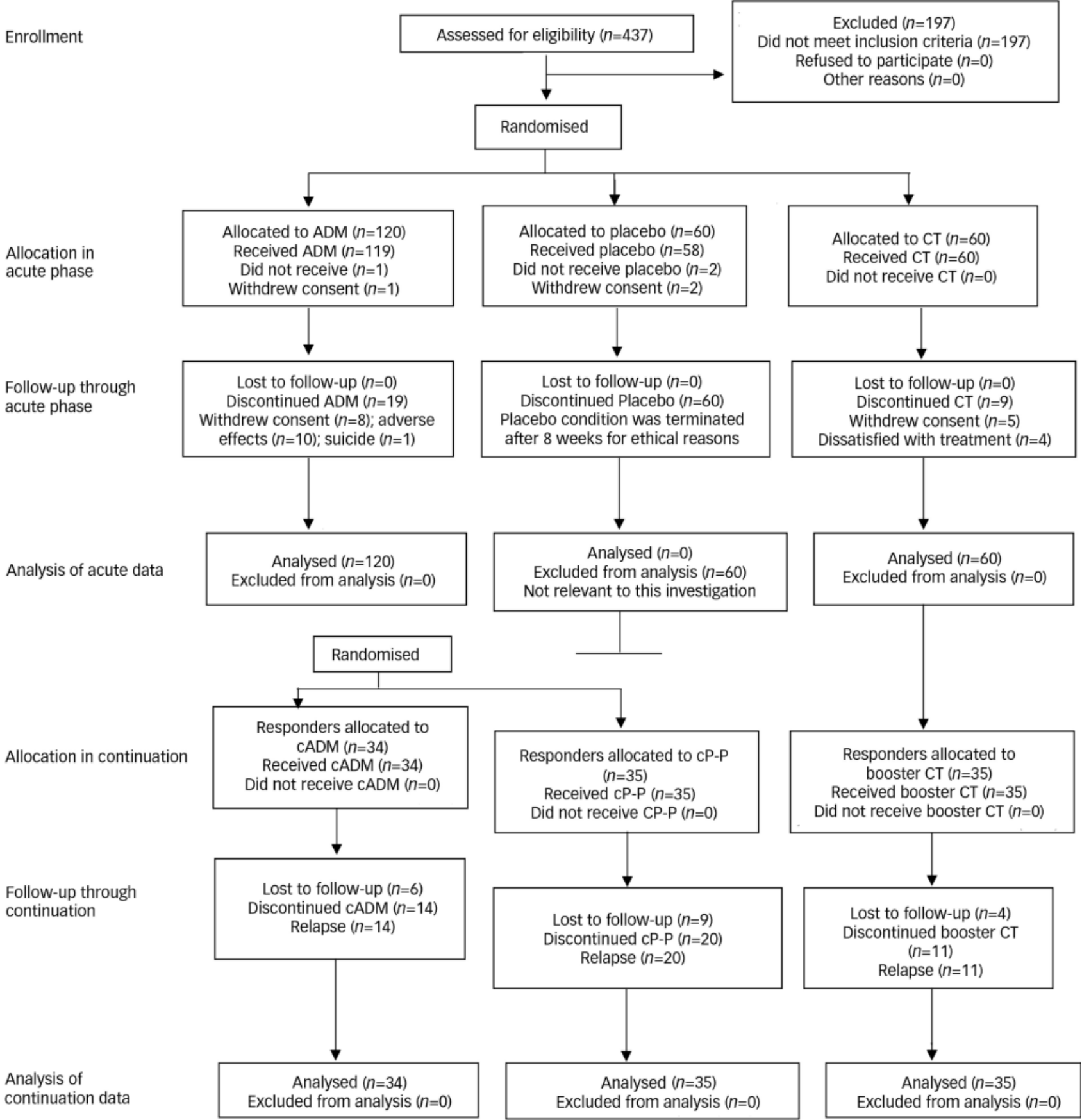

Fig. 1 Flow diagram of participants through the study. ADM, antidepressant medications; CT, cognitive therapy; CADM, continuation antidepressant medications during the continuation phase; CP-P, placebo during the continuation phase.

comparisons that include the placebo group were not included in the analyses below.

The criterion for response at 16 weeks was an HRSD score of 12 or lower. In order to limit the effect of transient mood fluctuations on the response designation, additional constraints required participants to have scored 14 or less at week 14 , or 12 or less at weeks 10 and 12. Individuals who scored higher than 12 at week 16 were still considered 'responders' if they had scored 12 or below at weeks 12 and 14, and again scored 12 or below at an additional evaluation at 18 weeks. Response at 16 weeks also required the completion of acute treatment.

Of the 180 people assigned to antidepressants or cognitive therapy, 104 people met the response criteria at 16 weeks and were entered into the 12-month continuation phase of the study. Half of the responders from the antidepressant group $(n=35)$ were randomly assigned to be withdrawn from medication onto pill placebo (continuation placebo) while the other half $(n=34)$ were continued on medication (continuation medication) throughout the 1-year continuation phase. In the cognitive therapy condition, regular therapeutic contact ceased at the end of the acute phase of treatment. Treatment responders $(n=35)$ could use up to three 1-hour booster sessions throughout the 1-year continuation phase. When individuals on antidepressants were randomised to continuation placebo or continuation medication at the beginning of the continuation phase, the process was triple blind - participants, pharmacotherapists 
and evaluators did not know which individuals were receiving active medication.

\section{Outcome measures}

The primary outcome measure was the 17 -item version of the HRSD. During the acute phase, assessments with evaluators masked to treatment condition were held weekly for the first 4 weeks and biweekly from week 6 to week 16. During the continuation phase, assessments were conducted during each of the first 2 weeks, biweekly through to the end of the second month, and monthly thereafter. Relapse was defined as a score of 14 or greater on the HRSD during two consecutive weeks (ad hoc assessments were scheduled as needed to confirm this temporal component). If a person experienced a worsening of symptoms in the interval between assessments, the timing of relapse was ascertained using the Longitudinal Interval Follow-up Evaluation, ${ }^{13}$ conducted at the next assessment. Of the 45 participants who were judged to have relapsed, 11 were ascertained using this instrument.

\section{Statistical analyses}

The primary statistical analyses examined whether there were differences in efficacy between the two treatments as a function of personality disorder status, by examining:

(a) the percentage of individuals meeting response criteria;

(b) change in average depression severity scores;

(c) the percentage who met response criteria and did not relapse post-treatment (sustained response).

Data from all participants randomised to treatment were included in all three sets of (intent-to-treat) analyses. In the case of attrition, all data collected up to the date of attrition were included. In each analysis, the treatment $\times$ personality disorder status interaction term was of primary interest.

For the acute phase data, Cochran-Mantel-Haenszel tests were used to assess differential response/non-response as a function of treatment and personality disorder status, stratified across the two sites. ${ }^{14}$ Odds ratios (ORs), confidence interval (CI) bands and interaction effects were assessed using a logistic regression model based on the likelihood ratio $\chi^{2}$ statistic. ${ }^{15}$ Continuous data were examined with multiple regression techniques using a last observation carried forward approach and with hierarchical linear modeling. The hierarchical linear modeling approach adjusts for repeated measures with nested random effects. ${ }^{16,17}$ Using this approach, each person's growth curve and true HRSD score at the end of treatment can be estimated from a collection of individual-specific parameters. ${ }^{18}$ For all hierarchical linear modeling analyses reported, an unstructured covariance structure was assumed in order to model random intercepts and slopes. Two baseline scores were obtained for all participants, allowing for a full intent-to-treat analysis while at the same time covarying for each person's initial baseline depression severity score. All models were performed using SAS Version 9.0 for Windows, PROC MIXED for hierarchical linear modelling analyses, PROC GLM for multiple regression analyses, PROC FREQ for CochranMantel-Haenszel tests, and PROC GENMOD for Logistic Regression (SAS Institute Inc, Cary, North Carolina, USA).

The Cox proportional hazards model was used to estimate attrition rates and relapse rates. ${ }^{19}$ Because a differential response rate for people with and without personality disorder emerged between the two treatments (see below), the Cox proportional hazards technique is inappropriate on its own to estimate survival rates during the continuation phase. Any indication of differential relapse might be an artifact of a differential sieve through which individuals who would be at greatest relapse risk already had failed to respond to a particular treatment, ${ }^{20}$ and therefore did not enter the continuation phase. To address this concern, estimated survival curves from Cox proportional hazards regression models were weighted by the proportion of people who responded in each treatment arm. This procedure estimates the percentage of people who both responded to treatment and maintained that response throughout the 1-year continuation phase. In the original publication, four possibly confounding covariates (dysthymia, atypical depression, number of prior episodes and gender) were entered in the survival analyses; ${ }^{8}$ these were performed using the present models as well. These models were performed using the SAS procedure, PROC PHREG.

\section{Results}

\section{Attrition}

The overall rate of participant attrition was detailed in the original publication. ${ }^{7}$ Although survival analyses revealed no overall difference between people with and without personality disorder $\left(\chi_{1}^{2}=0.32, P=0.57\right)$, a statistically non-significant trend-level personality disorder status $\times$ treatment interaction did emerge $\left(\chi_{1}^{2}=2.72, P<0.10\right)$. This effect was driven in large part by the fact that the attrition rate was lower for people with personality disorder $(12 \%)$ than for people without personality disorder $(21 \%)$ in the antidepressant arm, and lower for people without personality disorder $(12 \%)$ than for people with personality disorder $(22 \%)$ in the cognitive therapy arm.

\section{Outcome of acute treatment}

\section{Categorical response analyses}

There was a significant interaction between treatment and personality disorder status in acute treatment response $\left(\chi_{1}^{2}=6.77\right.$, $P=0.009)$. As displayed by the narrow bars in Fig. 2, people with personality disorder showed a non-significant trend in favour of antidepressants; 66\% (95\% CI 54-78) met response criteria compared with $44 \%$ (95\% CI 25-63) in the cognitive therapy arm (Cochran-Mantel-Haenszel $\chi_{1}{ }_{1}=3.42, P=0.06$; OR=2.42, 95\% CI $0.96-6.28)$. The reverse pattern was observed, as a non-significant trend, for people without personality disorder, with $70 \%$ (95\% CI 54-86) meeting response criteria in the cognitive therapy arm, compared with $49 \%$ (95\% CI 36-62) in the antidepressant arm (Cochran-Mantel-Haenszel $\chi_{1}^{2}=3.20, P=0.07$; OR=2.28, 95\% CI 0.94-5.81). The tests of the main effects of treatment, personality disorder status and the three-way site $\times$ treatment $\times$ personality disorder status interaction were non-significant (all $\chi^{2} s<0.43$, all $P s>0.51)$.

\section{Continuous response analyses}

In the original publication of the treatment outcome data, the authors reported a significant site $\times$ treatment interaction in the hierarchical linear modelling analyses; ${ }^{7}$ this interaction term was added to the models described below. Controlling for initial depression severity, the test of the treatment $\times$ personality disorder status interaction on estimates of week 16 HRSD scores was significant in the last observation carried forward $\left(\mathrm{F}_{1,173}=4.18, P=0.04\right)$ and hierarchical linear modeling analyses $\left(\mathrm{F}_{1,173}=4.32, P=0.04\right.$; Cohen's $d=0.76$ (95\% CI $0.32-1.20)$ ). Follow-up hierarchical linear modelling analyses among people with and without personality disorder yielded a non-significant trend in favour of antidepressants for people with personality disorder $\left(t_{173}=-1.69\right.$, $P=0.09)$ and a non-significant treatment effect in people without personality disorder $\left(t_{173}=1.24, \quad P=0.22\right)$. The test of the 


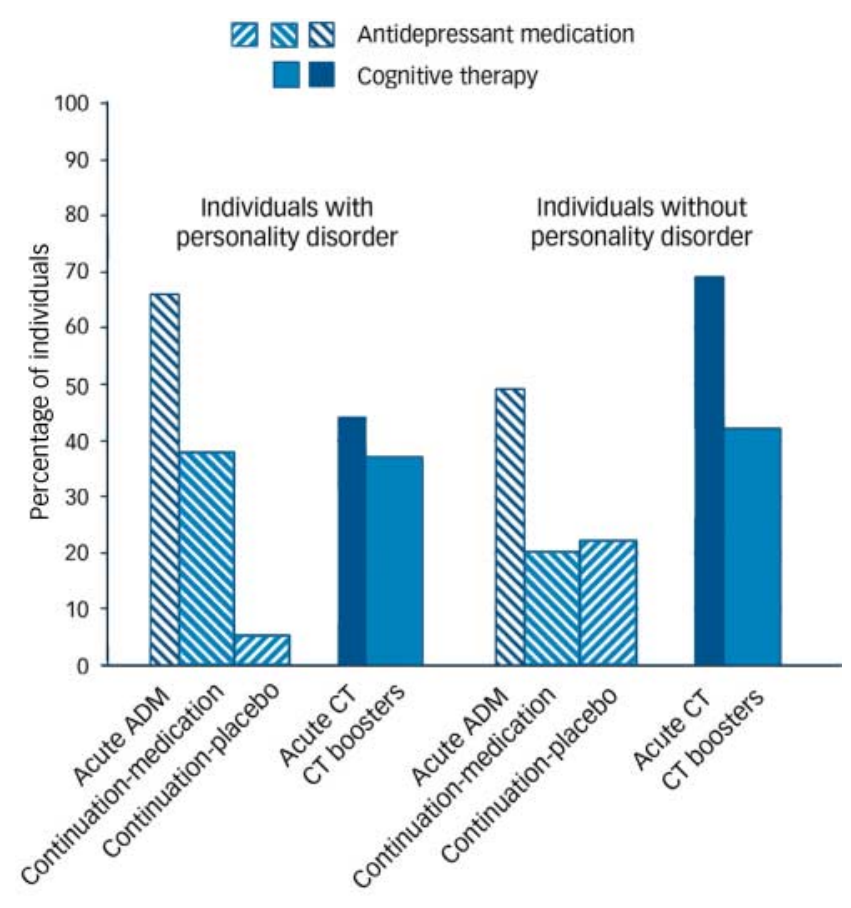

Fig. 2 The percentage of individuals in each treatment arm meeting response and sustained response criteria.

ADM, antidepressant medication; $\mathrm{CT}$, cognitive therapy. The narrow bars display the proportion of people who met response criteria in the two display the proportion of people who met response criteria in the two
treatments (antidepressants and cognitive therapy) at the end of the 16-week acute phase. The wide bars represent the estimated proportion of individuals who acute phase. The wide bars represent the estimated proportion of individuals who
survived the 12-month follow-up period without a relapse. Bars on the left half survived the 12-month follow-up period without a relapse. Bars on the left half
of the figure represent individuals diagnosed with a comorbid personality disorder; of the figure represent individuals diagnosed with a comorbid personality diso
bars on the right represent individuals who did not have a comorbid Axis-II diagnosis.

treatment $\times$ personality disorder status interaction on the linear slope estimates of symptom change, though in the same direction as those obtained in the last observation carried forward and hierarchical linear modelling intercept analyses, was not significant $\left(F_{1,171}=1.98, P=0.16\right.$; Cohen's $d=0.40$ ( $95 \%$ CI -0.04 to 0.84$)$ ).

\section{Potential confounds}

To address the possibility that people with personality disorder differed from those without personality pathology in other important ways, we examined eight history of illness variables, two depression subtype variables, four composite Axis I comorbidity variables, seven demographic variables and, for people in the antidepressant arm, dosage and augmentation, to determine whether people with and without personality disorder differed at the $P<0.10$ level. As shown in online table DS2, nine of these variables differed between the two groups. All nine variables were entered simultaneously into each of the models reported above. For the model predicting categorical response, as well as the hierarchical linear model predicting end of treatment HRSD scores, the personality disorder status $\times$ treatment interaction remained significant even with the simultaneous addition of all nine variables $\left(\chi^{2}{ }_{1}=5.59, P=0.02\right.$ for the categorical analysis; $F_{1,164}=4.13, P=0.04$ for the hierarchical linear model). In the last observation carried forward analysis, the personality disorder status $\times$ treatment interaction became a non-significant trend $\left(F_{1,164}=3.54, P=0.06\right)$.

\section{Individual personality disorder diagnoses}

The results reported thus far were achieved by comparing all participants with at least one personality disorder diagnosis with participants with no personality disorder diagnosis. In order to better determine what was driving the observed effects, we conducted exploratory analyses examining the relations for individual personality disorders. The strongest pattern occurred for people diagnosed with personality disorder not otherwise specified (NOS), wherein 12 of 16 people (75\%) responded to antidepressants and 3 of $9(33 \%)$ responded to cognitive therapy $(\mathrm{OR}=6.09$, 95\% CI 1.09-42.87, in favour of antidepressants over cognitive therapy). In order to examine this effect further, we assigned individuals diagnosed with personality disorder NOS to the personality disorder cluster in which their highest concentration of symptoms was observed. For example, a broad-band Cluster C category was formed by combining individuals with personality disorder NOS for whom the highest concentration of symptoms fell in Cluster $\mathrm{C}$ with those individuals who actually received a Cluster C diagnosis. The effect of personality disorder status was the strongest for individuals in the broad-band Cluster B category. Of the 16 people in the broad-band Cluster B grouping, 6 of the 9 individuals treated with antidepressants responded, whereas only 1 of 7 individuals being treated with cognitive therapy responded ( $\mathrm{OR}=15.03$, 95\% CI 1.36-515.92). For people in the broad-band Cluster C group, 32 of 47 (68\%) responded to antidepressants, whereas 10 of 19 (53\%) responded to cognitive therapy (OR=1.97, 95\% CI 0.65-5.98). For those in the broad-band Cluster A group, 2 of 4 responded to cognitive therapy $v$. 1 of 4 who responded to antidepressants $(\mathrm{OR}=3.28,95 \%$ CI $0.16-$ 137.50).

\section{Sustained response through the 12-month continuation phase}

For the 12-month continuation phase, the survival rates of the three treatment arms (prior cognitive therapy, continuation placebo and continuation medication) were estimated for individuals with and without personality disorder. Sustained response estimates for each group were then calculated by computing the product of these survival estimates and the group's treatment response rate (e.g. for people with personality disorder who had received antidepressant treatment, survival estimates for both the continuation medication and continuation placebo arms were multiplied by the percentage of people with personality disorder who responded to acute antidepressant treatment). Analysis of these estimates revealed a significant treatment $\times$ personality disorder status interaction in the percentage of people who showed a sustained response through the end of the 12 months $\left(\chi_{2}^{2}=6.13\right.$, $P=0.047)$. For people with personality disorder, a significant main effect of treatment emerged $\left(\chi_{2}^{2}=11.94, P=0.003\right)$. The wide bars in Fig. 2 show that despite the fact that a higher percentage of people with personality disorder responded to treatment (and hence entered the continuation phase) in the antidepressant arm (66\%) compared with the cognitive therapy arm (44\%), an estimated $38 \%$ of patients (95\% CI 20-56) initially randomised to cognitive therapy evidenced sustained response. With a higher relapse rate in the continuation medication arm, a nearly identical estimate of the proportion of people with sustained response was obtained in this group (38\% (95\% CI 21-55)). People with personality disorder who had previously received antidepressants but were withdrawn onto continuation placebo tended to relapse at an extremely high rate. Only $6 \%$ of these people ( $95 \%$ CI -3 to 15 ) exhibited sustained response. Specific contrasts revealed that, for people with personality disorder, prior cognitive therapy and continuation medication were each superior to continuation placebo on the sustained response variable $\left(\chi_{1}^{2}=9.80, P=0.002\right.$ for continuation medication $v$. continuation placebo; $\chi_{1}{ }_{1}=9.15, P=0.003$ for prior cognitive therapy $v$. continuation placebo). There was no difference in estimated 
sustained response rates between prior cognitive therapy and continuation medication for this group $\left(\chi_{1}^{2}=0.002, P=0.97\right)$.

For people without personality disorder, the main effect of treatment was not significant in the analysis of sustained response $\left(\chi_{1}^{2}=4.54, P=0.103\right)$. Figure 2 shows that for people initially randomised to receive cognitive therapy, 43\% (95\% CI 26-60) exhibited sustained response compared with $23 \%$ (95\% CI 8-38) for those initially randomised to receive antidepressant treatment who were then assigned to continuation placebo, and 21\% (95\% CI 7-35) for those who were assigned to antidepressant treatment and then were assigned to continuation medication.

\section{Discussion}

The primary purpose of this investigation was to determine whether personality pathology predicts differential response to two generally effective treatments for depression. In this sample of people with moderate-to-severe depression, short-term cognitive monotherapy proved relatively ill-suited for those with comorbid personality disorder, a pattern consistent with findings from the Nottingham Study of Neurotic Disorder in which cognitive therapy and self-help treatment were less effective for people with comorbid personality disorder than for those without Axis II pathology. ${ }^{21}$ Indeed, in the present study, fewer than half of the people with personality disorder responded to cognitive therapy. Those who did respond, however, tended to sustain their response throughout the ensuing 12-month continuation phase.

Antidepressants, on the other hand, worked particularly well to reduce depressive symptoms for patients diagnosed with comorbid personality pathology. Although individuals with and without personality disorder differed in several respects, including the incidence of comorbid anxiety disorders, none of these factors accounted statistically for the differential treatment effects. Results from the continuation phase of the study further support the conclusion that antidepressants had potent effects in this group in that nearly all of the people with personality disorder who were withdrawn from medication relapsed. For people with depression who did not have diagnosed personality pathology, there was a suggestion in the data that cognitive therapy was particularly effective at reducing depressive symptoms, relative to antidepressants.

\section{Limitations}

The pattern of findings in regard to acute treatment response was consistent across the three different analytical methods, and the test of significance for the interaction of interest was met in all three. However, in the analysis that employed hierarchical linear modelling, the interaction of interest was significant only in the prediction of 16-week scores and not in the prediction of symptom change over time. This inconsistency may have arisen because of the fact that hierarchical linear modelling analyses can lead to misleading estimates of improvement rates in data-sets containing non-random attrition. ${ }^{22,23}$ Consistent with the observation that people with personality disorder assigned to cognitive therapy and people without personality disorder assigned to antidepressants fared more poorly than the other two groups, individuals within these groups also tended to have higher rates of attrition. This is another indication that antidepressants were better-suited to people with personality disorder in this trial and that cognitive therapy was better suited to people without personality disorder. In addition, between-treatment differences in outcome within the subgroups of individuals with and without personality disorder were at the level of non-significant trends. Since these tests were associated with relatively low power, these comparisons will be most useful to the field as constituents of future meta-analyses that will combine the current findings with attempts to replicate these results.

A second limitation involves the duration of the active treatments. The differences between cognitive therapy and antidepressants in relation to personality disorder status may have depended, in part, on the fact that the treatments were brief, relative to the durations recommended for treating people with depression who have comorbid personality disorder. Given more time, cognitive therapy might have been as effective as antidepressants for people with personality disorders, and antidepressants might have been as effective as cognitive therapy for people without personality disorders. Nevertheless, short-term treatment did lead to response percentages of up to two-thirds of people with personality disorder in the antidepressant arm, as well as for people without personality disorder in cognitive therapy.

Third, prospective participants with diagnosed schizotypal, antisocial or borderline personality disorders were excluded from the trial. Thus, these results do not, strictly speaking, generalise to the population that includes people with these comorbidities. However, only $5 \%$ of otherwise eligible participants were excluded because they received one of these diagnoses, and all but one of these individuals was excluded because of a Cluster B diagnosis. People in this trial whose highest concentration of personality disorder symptoms occurred in Cluster B showed a particularly strong response to antidepressants relative to cognitive therapy. This suggests that the pattern we observed might be even stronger in a sample that includes all Axis II comorbidities.

\section{Future directions}

Because the primary medication used in this study, paroxetine, has demonstrated efficacy in treating depression with comorbid anxiety disorders, it might have been expected that the antidepressants' relative efficacy for people with personality disorder was due to its superiority for patients with Cluster $\mathrm{C}$ personality disorders. ${ }^{24}$ However, the numerical advantage for antidepressants was strongest for people with Cluster B personality pathology. One possible explanation for this result is suggested by research demonstrating that people with Cluster B personality disorders display deficits in cortical regions thought to underlie the regulation of emotion and the inhibition of impulsive aggression, ${ }^{25}$ and from studies that demonstrate that selective serotonin reuptake inhibitors can reduce mood lability, anger and impulsive behaviour in humans and animals. ${ }^{26-28}$ Future studies might attempt to further investigate these mechanisms in people with depression and this type of personality pathology. In addition, the surprisingly high rate of relapse among people with personality disorder withdrawn from medication should be examined in future research, as the mechanism driving this effect is currently unclear.

\section{Clinical implications}

The pattern of results from this study suggests possible prescriptive recommendations regarding short-term treatment for people with moderate-to-severe depression as a function of comorbid personality pathology. Evidence from this study, consistent with findings from other investigations, suggests that for people with depression with a comorbid personality disorder, paroxetine treatment is more likely than cognitive therapy to alleviate their depressive symptoms in the short term. Because of a higher relapse rate for people receiving continuation medication, shortterm cognitive therapy may produce rates of sustained response roughly equivalent to those achieved with sustained antidepressants, provided that booster sessions are given to individuals receiving cognitive therapy. Given the relatively low relapse rate 
among people with personality disorder who responded to cognitive therapy, the combination of antidepressants and cognitive therapy might be especially valuable for these people. Individuals without a personality disorder diagnosis fared better during acute treatment and exhibited a higher sustained response rate while receiving cognitive therapy, compared with antidepressants. Indeed, these people appeared to be equally susceptible to relapse following acute treatment with antidepressants regardless of whether they were continued on medication or withdrawn onto placebo. This pattern, if replicated, could lead to the consideration of cognitive therapy as a first-line treatment for people with depression who do not have a personality disorder.

Jay C. Fournier, MA, Robert J. DeRubeis, PhD, Department of Psychology, University of Pennsylvania, Philadelphia, Pennsylvania; Richard C. Shelton, MD, Department of Psychiatry, Vanderbilt University, Nashville, Tennessee; Robert Gallop, PhD, Department of Mathematics and Applied Statistics, West Chester University, West Chester, Pennsylvania; Jay D. Amsterdam, MD, Department of Psychiatry, University of Pennsylvania, Philadelphia, Pennsylvania; Steven D. Hollon PhD, Department of Psychology, Vanderbilt University, Nashville, Tennessee, USA

Correspondence: Robert J. DeRubeis, Department of Psychology, University of Pennsylvania, Philadelphia, PA 19104-6196, USA. Email: derubeis@psych.upenn.edu

First received 21 Feb 2007, final revision 16 Oct 2007, accepted 29 Oct 2007

\section{Acknowledgements}

We thank our colleagues for contributing to this research. J.C.F. drafted the manuscript and conducted the statistical analyses. R.J.D. and S.D.H. were the principal investigators and oversaw the implementation of cognitive therapy at the respective sites J.D.A and RCS. were the CO-principal investigators and supervised the implementation of medication treatment. R. provided methodological and statistical expertise. All authors had full access to all of the data in the study and take responsibility for the integrity of the data and the accuracy of the data analysis. We also thank our colleagues who helped make this research possible. Paula R. Young and Margaret L. Lovett served as the study coordinators. John P. O'Reardon, Ronald M. Salomon and the late Martin Szuba served as study pharmacotherapists (along with J.D.A and R.C.S.). Cory P. Newman, Karl N. Jannasch, Frances Shusman and Sandra Seidel served as the cognitive therapists (along with R.J.D. and Sokol provided consultation with respect to the implementation of cognitive therapy. Madeline M. Gladis and Kirsten L. Haman oversaw the training of the clinical interviewers, and David Appelbaum, Laurel L. Brown, Richard C. Carson, Barrie Franklin, Nana A. Landenberger, Jessica Londa-Jacobs, Julie L. Pickholtz, Pamela Fawcett-Pressman, Sabine Schmid, Ellen D. Stoddard, Michael Suminski and Dorothy Tucker served as project interviewers. Joyce L. Bell, Brent B. Freeman, Cara C. Grugan, Nathaniel R. Herr, Mary B. Hooper, Miriam Hundert, Veni Linos and Tynya Patton provided research support. This research was supported by grants MH50129 (R10), MH55875 (R10), MH01697 (K02) and MH01741 (K24) from the National Institute of Mental Health, Bethesda, Maryland, USA. GlaxoSmithKline provided medications and pill placebos for the trial.

\section{References}

1 American Psychiatric Association. Practice guideline for the treatment of patients with major depressive disorder (revision). Am J Psychiatry 2000; 157: 49.

2 Elkin I, Shea MT, Watkins JT, Imber SD, Sotsky SM, Collins JF, Glass DR, Pilkonis PA, Leber WR, Docherty JP. National Institute of Mental Health Treatment of Depression Collaborative Research Program: General effectiveness of treatments. Arch Gen Psychiatry 1989; 46: 971-82.

3 Hollon SD, Jarrett RB, Nierenberg AA, Thase ME, Trivedi M, Rush AJ. Psychotherapy and medication in the treatment of adult and geriatric depression: which monotherapy or combined treatment? J Clin Psychiatry 2005; 66: 455-68.

4 Hardy GE, Barkham M, Shapiro DA, Stiles WB, Rees A, Reynolds S. Impact of Cluster $C$ personality disorders on outcomes of contrasting brief psychotherapies for depression. J Consult Clin Psychol 1995; 63: 997-1004.

5 Kool S, Schoevers R, de Maat S, Van R, Molenaar P, Vink A, Dekker J. Efficacy of pharmacotherapy in depressed patients with and without personality disorders: A systematic review and meta-analysis. J Affect Disord 2005; 88: 269-78.
6 Newton-Howes G, Tyrer P, Johnson T. Personality disorder and the outcome of depression: meta-analysis of published studies. Br J Psychiatry 2006; 188: $13-20$.

7 DeRubeis RJ, Hollon SD, Amsterdam JD, Shelton RC, Young PR, Salomon RM, O'Reardon JP, Lovett ML, Gladis MM, Brown LL, Gallop R. Cognitive therapy vs medications in the treatment of moderate to severe depression. Arch Gen Psychiatry 2005; 62: 409-16.

8 Hollon SD, DeRubeis RJ, Shelton RC, Amsterdam JD, Salomon RM, O'Reardon JP, Lovett ML, Young PR, Haman KL, Freeman BB, Gallop R. Prevention of relapse following cognitive therapy vs medications in moderate to severe depression. Arch Gen Psychiatry 2005; 62: 417-22.

9 First M, Spitzer R, Gibbon M, Williams J. Structured Clinical Interview for DSM-IV-TR - Axis I Disorders, Research Version, Patient Edition with Psychotic Screen (SCID-I/P W/ PSY SCREEN). Biometrics Research, New York State Psychiatric Institute, 2001.

10 Hamilton M. A rating scale for depression. J Neurol Neurosurg Psychiatry 1960; 23: 56-62.

11 Spitzer R, Williams J, Gibbon M, First M. Structured Clinical Interview for DSM-II-R Personality Disorders (SCID-II, Version 1.0). American Psychiatric Press, 1990.

12 Doyle TJ, Tsuang MT, Lyons MJ. Comorbidity of depressive illnesses and personality disorders. In Comorbidity in Affective Disorders (ed M Tohen): 105-156. Marcel Dekker Inc., 1999

13 Keller MB, Lavori PW, Friedman B, Nielsen E, Endicott J, McDonald-Scott P, Andreasen NC. The Longitudinal Interval Follow-up Evaluation. A comprehensive method for assessing outcome in prospective longitudinal studies. Arch Gen Psychiatry 1987; 44: 540-8.

14 Kuritz S, Landis J, Koch G. A General overview of Mantel-Haenszel methods: applications and recent developments. Ann Rev Public Health 1988; 9: 12360.

15 Hosmer D, Lemeshow S. Applied Logistic Regression. Wiley, 1989.

16 Bryk A, Raudenbush S. Hierarchical Linear Modeling: Applications and Data Analysis Methods. Sage, 1996.

17 Goldstein H. Models in Educational and Social Research. Oxford University Press, 1987.

18 Willett JB. Measuring change: What individual growth modeling buys you. In Change and Development: Issues of Theory, Method, and Application. The Jean Piaget Symposium Series (eds E Amsel, KA Renninger): 213-243. Lawrence Erlbaum Associates, 1997.

19 Cox D, Oakes D. Analysis of Survival Data. Chapman \& Hall, 1984.

20 Klein DF. Preventing hung juries about therapy studies. J Consult Clin Psychol 1996; 64: 81-7

21 Tyrer $P$, Seivewright N, Ferguson B, Murphy S, Johnson AL.The Nottingham study of neurotic disorder. Effect of personality status on response to drug treatment, cognitive therapy and self-help over two years. $\mathrm{Br} J$ Psychiatry 1993; 162: 219-26.

22 Diggle $\mathrm{P}$, Kenward MG. Informative drop-out in longitudinal data analysis. Appl Stat 1994; 43: 49-93.

23 Gibbons RD, Hedeker D, Elkin I, Waternaux C, Kraemer HC, Greenhouse JB, Shea MT, Imber SD, Sotsky SM, Watkins JT. Some conceptual and statistical issues in analysis of longitudinal psychiatric data: Application to the NIMH Treatment of Depression Collaborative Research Program dataset. Arch Gen Psychiatry 1993; 50: 739-50.

24 Rouillon F. Depression comorbid with anxiety or medical illness: The role of paroxetine. Int J Psychiatry Clin Pract 2001; 5: 3-10.

25 Johnson PA, Hurley RA, Benkelfat C, Herpertz SC, Taber KH. Understanding emotion regulation in borderline personality disorder: contributions of neuroimaging. J Neuropsychiatry Clin Neurosci 2003; 15: 397-402.

26 New AS, Buchsbaum MS, Hazlett EA, Goodman M, Koenigsberg HW, Lo J, Iskander L, Newmark R, Brand J, O'Flynn K, Siever L. Fluoxetine increases relative metabolic rate in prefrontal cortex in impulsive aggression. Psychopharmacology 2004; 176: 451-8.

27 Salzman C, Wolfson AN, Schatzberg A, Looper J, Henke R, Albanese M, Schwartz J, Miyawaki E. Effect of fluoxetine on anger in symptomatic volunteers with borderline personality disorder. J Clin Psychopharmacol 1995; 15: 23-9.

28 Wolff MC, Leander D. Selective serotonin reuptake inhibitors decrease impulsive behavior as measured by an adjusting delay procedure in the pigeon. Neuropsychopharmacology 2002; 27: 421-9. 